4. Il n'est possible d'apprécier le " colititre " d'un lait d'après le taux des nitrites, que seulement dans le cas où les coliformes constituent la majorité des microbes du lait examiné.

5. L'allure de la réaction de la nitroréductase donne précisément la possibilité de prouver si, dans le lait examiné, les coliformes constituent ou non la majorité des microbes du lait.

6. L'épreuve de la nitroréductase est une méthode plus sûre et plus rapide que celle de la réductase.

\title{
BIBLIOGRAPHIE
}

[1] C. G. Macris et C. G. Tzivanopoulos. Contribution au contrôle bactériologique du lait. Agrotiki Epithéorissis (en langue greoque), juilletaoût, 1949.

[2] Journal of Dairy Science, 1935, 18, 705.

[3] A. Rochaix et A. Tapernoux. Le lait et ses dérívés, Paris-Lyon, 1948.

\section{DÉTERMINATION DE LA TENEUR \\ EN MATIËRE GRASSE DES FROMAGES, AU MOYEN DU BUTYROMÈTRE GERBER (1)}

$\begin{array}{ccc}\text { A. GRANVILLE } & \text { par } \\ \begin{array}{c}\text { Chargé de cours à l'Ecole } \\ \text { de Médecine vétérinaire, } \\ \text { à Cureghem }\end{array} & \text { et } & \text { F. DESMET } \\ & & \text { Ingénieur des Industries Agricoles } \\ \text { Chimiste au Laboratoire Inter- } & \text { A.l.Gx } \\ \text { communal de Bruxelles }\end{array}$

La simplicité de la méthode Gerber, pour la détermination de la matière grasse dans le lait, nous a conduits à essayer d'en étendre l'application à d'autres produits laitiers, notamment aux fromages.

La méthode acido-butyrométrique de dosage de la matière grasse dans les fromages n'est certes pas neuve, mais jusqu'à présent on utilisait les butyromètres spéciaux de Van Gulik. Ce, procédé présente un inconvénient: la dissolution du fromage logé dans un petit godet perforé, placé dans l'ouverture d'un bouchon en caoutchouc, est parfois laborieuse. Il nous est arrivé maintes fois de devoir faire séjourner pendant plusieurs heures au bainmarie, même en procédant à de fréquentes agitations, des fromages à pâte dure tels que Gruyère, Chester, Hollande, etc.

Il nous a done paru intéressant d'étudier un procédé se basant sur les mêmes principes, mais d'application plus simple et surtout plus rapide.

(1) Annales de Médecine Vétérinaire (Bruxelles), 1951, n' 1, 31. 
Nous avons utilisé le butyromètre à lait ordinaire, tel qu'il est actuellement défini par la législation belge.

Nous pesons environ 2 grammes de fromage sur un morceau de cellophane taré, nous enroulons le fromage et la cellophane à la manière d'une cigarette, et nous l'introduisons dans un butyromètre ordinaire contenant 10 millilitres $\mathrm{H}^{2} \mathrm{SO}^{4}$ pour Gerber. Nous ajoutons une quantité d'eau pour complèter la prise à 11 grammes, puis 1 millilitre d'alcool amylique. Le butyromètre est bouché et agité pour bien dissoudre le fromage. La dissolution est immédiate pour les fromages frais, les Brie, Camembert, fondus, etc. De toute façon, et afin d'éviter tout mécompte, nous plaçons le butyromètre dans un bain-marie dont la température est de 65 à $68^{\circ}$, et nous l'y maintenons pendant un quart d'heure en agitant fréquemment Après une centrifugation (10 minutes), nous plongeons le butyromètre au bain-marie pendant une dizaine de minutes et effectuons la lecture.

La teneur en matière grasse est obtenue par la formule :

$$
\text { M. G. } \%=\frac{\text { lecture } \times 11}{\text { prise d'essai }}
$$

Nous insistons vivement sur la question de la température du bain-marie; elle doit être scrupuleusement observée si l'on veut obtenir de bons résultats.

Nous avons comparé les résultats obtenus avec ceux que donne le procédé Van Gulik sans correction de lecture.

On trouvera dans le tableau ci-dessous les résultats comparatifs des deux méthodes.

\begin{tabular}{|c|c|c|c|}
\hline Variétés de fromages & $\begin{array}{l}\text { Méthode } \\
\text { Gerber } \\
\text { (1) }\end{array}$ & $\begin{array}{c}\text { Méthode } \\
\text { Van Gulik } \\
\text { (2) }\end{array}$ & Remarque \\
\hline$\ldots \ldots \ldots \ldots$ & 18,6 & 18,5 & Moyenne de 12 analyses \\
\hline Gouda $\ldots \ldots \ldots \ldots$ & 22,4 & 22,6 & 1 échantillon \\
\hline Hollande . . . . . . . . & 32,8 & 33,0 & 1 échantillon \\
\hline Edam gras .......... & 32,4 & 32,4 & 1 échantillon \\
\hline Herve ........... & 26,2 & 25,8 & 1 échantillon \\
\hline Roquefort $\ldots \ldots \ldots$ & 33,2 & 33,1 & 1 échantillon \\
\hline Herve fondu........ & 24,8 & 25,1 & 1 échantillon \\
\hline Fondus $\ldots \ldots \ldots \ldots$ & 21,6 . & 21,6 & 4 échantillons \\
\hline Fromages frais ...... & 17,8 & 17,8 & 2 échantillons \\
\hline
\end{tabular}

On peut done déduire que les deux méthodes acido-butyrométriques donnent des résultats sensiblement équivalents. 
Nous en étions arrivés là dans nos déterminations quand parurent trois publications qui nous orientèrent dans une nouvelle direction.

P. Macherel [1], dans un historique des méthodes acidobutyrométriques du dosage de la matière grasse dans les crèmes, émit lé vœu de voir étudier d'une façon plus approfondie les mêmes méthodes appliquées au lait et aux fromages.

Et tandis que PIEN [2], reprenant une critique de la méthode internationale pour l'analyse des fromages, critique émise par M. Florentin [3], établit une comparaison entre la méthode Schmid-Bondzinsky-Ratzlaff et l'extraction directe qu'il prend comme référence, Bernatrts [4] examine les résultats obtenus par les méthodes Roeder et Van Gulik en comparaison avec la méthode Weibull, qu'il prend comme base.

Notons que précédemment, L. C. JANSE [5] avait déjà comparé le procédé Weibull dans le dosage de la matière grasse des produits laitiers par rapport aux méthodes Röse Gottlieb et Gerber.

Bernaerts et Janse obtiennent des résultats plus élevés par les procédés acido-butyrométriques que par. le Weibull, qu'ils prennent comme étalon. Pour le premier, la différence serait provoquée par la formation d'éthers et d'esthers d'amyle; pour le second, elle serait imputable à une erreur de graduation des butyromètres. JANSE propose d'ailleurs soit de ramener la contenance des pipettes à lait de 11 à $10 \mathrm{ml}$. 79 , ou de multiplier les résultats obtenus par le facteur 0,981 .

Nous avons procédé à une série de déterminations de matières grasses par les méthodes Weibull et notre méthode Gerber.

Dans chaque cas, le procédé Weibull a donné des résultats inférieurs à ceux du Gerber, mais on ne peut vraiment pas suivre JANSE dans ses conclusions, car la différence est parfois bien supérieure à celle qu'il indique.

Nous reprenons ci-après quelques résultats obtenus (voir tableau page suivante.

Le Weibull avait été effectué sur 50 millilitres de lait. Il semble donc que, contrairement à certaines affirmations, la méthode Gerber ne donne pas systématiquement des résultats supérieurs à ceux de la méthode Weibull.

Dans les produits frais, n'ayant pas subi de fermentation poussée, les résultats des deux méthodes sont comparables. Des différences apparaissent lorsque l'on traite des fromages fermentés, et on peut constater alors que ces différences sont loin d'être constantes.

Il est évident que lorsque la matière grasse a subi des modifications, quand il y a mise en liberté d'acides gras, certains passeront 


\begin{tabular}{|c|c|c|}
\hline Variétés de fromages & Méthode Gerber & Weibull \\
\hline 3 & & \\
\hline Gervais triple crème..$\ldots \ldots \ldots \ldots$ & 20,1 & 20,21 \\
\hline Gervais double erème ........... & 15,5 & 15,35 \\
\hline Gruyère fondu $\ldots \ldots \ldots \ldots \ldots \ldots$ & 26,7 & 26,60 \\
\hline Fromage blanc............... & 24,6 & 24,65 \\
\hline Brie frais. . . . . . . . . . . & 10,3 & 10,46 \\
\hline Gervais $\ldots \ldots \ldots \ldots \ldots \ldots \ldots \ldots$ & 17,3 & 17,32 \\
\hline Brie frais . . . . . . . . . . . . & 13,2 & 13,14 \\
\hline Brio frais $\ldots \ldots \ldots \ldots \ldots \ldots \ldots \ldots$ & 14,4 & 14,37 \\
\hline Gervais $\ldots \ldots \ldots \ldots \ldots \ldots \ldots$ & 21,2 & 20,85 \\
\hline 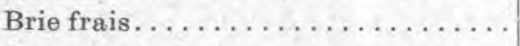 & 19,7 & 19,60 \\
\hline Roquefort $\ldots \ldots \ldots \ldots \ldots \ldots \ldots$ & 33,2 & 29,86 \\
\hline Bleu danois $\ldots \ldots \ldots \ldots \ldots \ldots \ldots$ & 36,7 & 34,58 \\
\hline 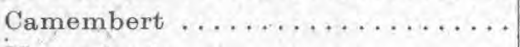 & 14,8 & 13,40 \\
\hline 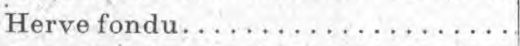 & 24,8 & 23,88 \\
\hline Brie avancé $\ldots \ldots \ldots \ldots \ldots \ldots \ldots$ & 19,5 & 18,02 \\
\hline \multicolumn{3}{|c|}{$\begin{array}{l}\text { D'autre part, deux essais comparatifs effectués avec du lait } \\
\text { frais nous ont donné : }\end{array}$} \\
\hline & I & II \\
\hline Gerber & 3,0 & 2,95 \\
\hline Weibull................... & 2,996 & 2,952 \\
\hline
\end{tabular}

dans le filtrat du Weibull, et dès lors cette méthode ne pourra plus donner des résultats exacts.

Là, pensons-nous, se trouve la cause principale des différences que l'on constate dans les résultats.

Nous avons enfin effectué une série d'essais comparatifs entre notre méthode Gerber et l'extraction directe.

Pour cette dernière, nous avons voulu éviter les manipulations indiquées par Pien, en utilisant l'extracteur Tchetcheroff en usage au Laboratoire Intereommunal.

Nous avons remplacé le sable par du sulfate de soude anhydre.

Après beaucoup de tâtonnements, nous avons finalement adopté le procédé suivant : 5 grammes de fromage sont broyés au mortier avec 30 grammes de sulfate; après une homogénéisation parfaite, le mortier est placé pendant trois à quatre heures à l'étuve, à $60-65^{\circ}$; après refroidissement, on broie encore convenablement la masse, et on l'introduit dans une cartouche d'extration. Le mortier est rincé au sulfate et à l'éther.

Le nombre de déterminations effectuées par ce procédé est encore trop faible pour permettre de tirer une conclusion définitive, mais nous pouvons cependant déjà affirmer que l'extraction directe et le Gerber donnent des résultats très voisins. 


\title{
BIBLIOGRAPHIE
}

[1] P. Macherel. Le Lait, 1948, 28, 256.

[2] J. PIEN. Le Eait, 1948, 28, 225.

[3] D. Florentin. Le Lait, 1939, 19, 10.

[4] M. BenNaerts. Nederl. Melk-Zuiveltijdschrift, 1948, 2, 99.

[5] L. C. JANSE. Methods of fat determination in dairy products. Chem. Weekblad, 1946, 42.

\section{INFLUENCE DES ALBUMINES SUR LA STABILITÉ DU BEURRE (1)}

\author{
par

\section{P. DIATCHENKO}

(Institut des recherches scientifiques de l'Industrie Laitière de l'U.R.S.S.)

Dans un article antérieur (Industrie Laitière, avril 1949) sur la teneur en albumines et la composition du plasma du beurre, nous avons montré que le goût et l'odeur du beurre dépendent dans une certaine mesure des propriétés du plasma.

Les albumines du plasma pendant leur conservation peuvent subir sous l'influence des microorganismes, une certaine dégradation. Celle-ci peut être la cause des défauts du gout du beurre. Le but de nos recherches est d'élucider cette question.

Dans les essais de laboratoire le plasma a été extrait du beurre de vache frais. Le liquide a été ensemencé ensuite par de différentes bactéries. Après un séjour de cinq jours dans un thermostat, on examinait le goût du plasma et déterminait le taux de dégradation des albumines en dosant l'azote aminé. Le tableau I montre que la décomposition des albumines du plasma détermine l'apparition des défauts suivants : goût amer, putrescent, etc.

Il est à signaler notamment que le goût dit " de poisson " apparaît dans le plasma lors d'une putréfaction profonde. Ceci est en contradiction avee l'explication admise généralement, selon laquelle l'origine de ce défaut serait le processus catalytique de décomposition de la lécithine sous l'action du sel et de l'acide lactique avec formation de la triméthýlamine: Nos essais ont montré que ce défaut peut apparaître lors de la dégradation microbiologique du complexe albumino-lécithinique des enveloppes des particules sphériques de la graisse.

(1) Industrie Laitière (de 1'U.R.S.S.), juillet 1949, p. 17-20. (Tracluction C. Barkovsky.) 